\title{
Evaluation of the bit error rate and Q-factor in optical networks
}

\author{
Tomáš Ivaniga ${ }^{1}$, Petr Ivaniga ${ }^{2}$ \\ ${ }^{l}$ (Department of Electronic and Multimedia Communications, Faculty of Electrical Engineering and \\ informatics/ University of Technology Košice, Košice, Slovakia) \\ ${ }^{2}$ (Department of Transportation Networks, Faculty of Management Science and Informatics/University of \\ Žilina, Žilina, Slovakia)
}

\begin{abstract}
The article defines the relation between the error parameters in networks that are built on optical transmission paths in relation to the optical wireless links. Due to the large volume of the transmitted data caused by the association of many tributary signals, the high demands on the transmission error are expected. The aim of the article is to develop measurement methods, evaluation of individual error parameters and explain the relation between them.
\end{abstract}

Keywords: Bit Error Ratio, Quality Factor.

\section{Introduction}

Development in backbone networks goes the way of fully optical network elements based on dense wavelength multiplexing of DWDM technology with a terabit capacity on a thousands of miles distance. The advantage of DWDM technology is the possibility of effective recovery and amplification of the signal, using a single erbium-doped fiber amplifier for all channels, which technologically enables the recovery and the gain for all channels simultaneously, and is convenient for the transmission in the core networks. Optical devices, permitting the transmission of such capacities, are complicated as they have to deal with a number of physical parameters such as a signal attenuation, noise, polarization dispersion, chromatic dispersion, non-linear interference and much more. This article aims to define the relation between the error parameters in optical networks in relation to the optical wireless links, which are using the atmosphere as a transmission medium for the propagation of optical beam. Optical fiber-free joints are sensitive to the state of the transmission environment and compared to the conventional optical link, the connection quality depends on the time of a day, air condition etc.

\section{Basic Definition Of The Bit Error Rate}

One of the main parameters describing the quality of the data link is a bit error rate BER (Bit Error Rate). With BER is possible to compare the quality of different systems for data transmission. Bit error rate is defined by the following equation,

$$
B E R=\frac{n_{\varepsilon}}{N_{B}}
$$
interval. [1]

where $n_{c}$ is the number of bits received in error and $N_{B}$ is the total number of bits received in the defined time

For modern transmission networks is used the information transfer in larger blocks called packets. The packet consists of a certain number of bits that can be selected or prescribed by the type of network. The occurrence of incorrectly transmitted bit causes degradation of the entire packet. In terms of the error rate, the large amount of data are lost. This error is determined by the relationship (2).

$$
P E R=\frac{N_{E R P}}{N_{P}}
$$

$N_{E R P_{-}}$number of transmitted packets with the occurrence of at least one incorrectly transmitted bit

$N_{p}$ - total number of transmitted packets

For optical fiber-free connections is defined significant error parameter and it is the relative time interval $p$, which expresses the percentage of the link downtime $t_{i}$ to the total time of operation $T_{c}$ according to equation (3). This parameter is based on the possibility of a connection failure due to atmospheric turbulence and fluctuation of received power. For reliable determination of this parameter for a particular connection must be selected sufficiently long period $T_{c}$. This is usually the period of one year.

$$
p=\frac{\sum_{i}^{\infty} t_{i}}{\tau_{c}} \cdot 100 \quad[\%, s, s]
$$

As it can be seen from the formula for determining the bit error rate (1), it is necessary to know the total number of transmitted bits $N_{B}$. This number can be determined by permanent monitoring of the number of transmitted bits. It is important to note that for example $B E R-10^{-12}$ corresponds to one erroneously transmitted bit attributable to $10^{12}$ bits transferred in total. At a communication speed of $155 \mathrm{Mbps}$ occurs one error in the transmission in average once every 6450 
$\mathrm{s}$, when the data speed is $2048 \mathrm{kbps}$ even once in $500000 \mathrm{~s}$. The bit error rate measurement corresponds with the character to a binomial probability distribution $P_{B I N}$ according to the following equation,

$$
P_{B I N}\left(n_{c}, N_{B} B E R\right)=\frac{N_{B} !}{\left(N_{B}-n_{c}\right) !} \cdot B E R^{n_{c}} \cdot(1-B E R)^{N_{B}-n_{c}}
$$

$\mathrm{P}_{\mathrm{BIN}}$ value expresses the occurrence probability of a certain number of errors $n_{c}$ to the total number of transmitted bits $N_{B}$ for the $B E R$. [2]

In case that the error rate is relatively low $\left(<10^{-4}\right)$, it is possible to use simpler Poisson distribution. To express the occurrence probability of a defined number of errors by the Poisson distribution, we had to introduce a parameter $\mu$, which expresses the probability of erroneous transmission of one bit. The parameter $\mu$ can be defined as follows.

$$
\mu=B E R . N_{B}
$$

With the relations (4), (5) and the $B E R$ value, we are able to determine the probability of the $n_{c}$ erroneous bits delegated in the total number of bits $N_{B}$. It is also possible to determine the total number of transmitted bits $N_{B}$ to the value of $B E R$ with the desired accuracy. Assuming that we consider only the appearance of one erroneously transferred bit $n_{e}=1$, and we require accuracy in determining the error $P_{P O I S S}\left(n_{e}, \mu\right)=0,99$, the minimum and maximum values of the parameter $\mu$ can be derived by numerical methods. For a known value of the parameter $B E R$, according to (5), we are also able to determine the number of transmitted bits $N_{B}$. From the total number of bits and transmitted rate $v_{i}$, we can determine the required measurement time $t_{m e r}$ by the following equation,

$$
t_{\text {mer }}=\frac{N_{B}}{v_{i}}
$$

where $v_{i}$ is the transfer speed in bps. The calculated minimum values of measurement time for determining the bit error rate are shown in Table 1.

Table 1 Measurement time for the transmission speed 2, $048 \mathrm{Mbps}$ in the range of $10^{-6}-10^{-14}$

\begin{tabular}{|c|c|c|c|c|}
\hline \multirow{2}{*}{$B E R$} & \multicolumn{2}{|c|}{$N_{B}(\mathrm{bit})$} & \multicolumn{2}{c|}{$t_{\text {mer }}$} \\
\cline { 2 - 5 } & min. & max. & min. & max. \\
\hline $10^{-14}$ & $1,49.10^{13}$ & $6,64.10^{14}$ & $84 \mathrm{~d} 06: 13: 20$ & $3750 \mathrm{~d}$ \\
\hline $10^{-13}$ & $1,49.10^{12}$ & $6,64.10^{13}$ & $8 \mathrm{~d} 10: 13: 20$ & $375 \mathrm{~d}$ \\
\hline $10^{-12}$ & $1,49.10^{11}$ & $6,64.10^{12}$ & $0 \mathrm{~d} 20: 13: 20$ & $37 \mathrm{~d} 12: 00: 00$ \\
\hline $10^{-11}$ & $1,49.10^{10}$ & $6,64.10^{11}$ & $0 \mathrm{~d} 02: 01: 20$ & $3 \mathrm{~d} 18: 00: 00$ \\
\hline $10^{-10}$ & $1,49.10^{9}$ & $6,64.10^{10}$ & $0 \mathrm{~d} 00: 12: 08$ & $0 \mathrm{~d} 09: 00: 00$ \\
\hline $10^{-9}$ & $1,49.10^{8}$ & $6,64.10^{9}$ & $0 \mathrm{~d} 00: 01: 13$ & $0 \mathrm{~d} 00: 54: 00$ \\
\hline $10^{-8}$ & $1,49.10^{7}$ & $6,64.10^{8}$ & $0 \mathrm{~d} 00: 00: 07$ & $0 \mathrm{~d} 00: 05: 24$ \\
\hline $10^{-7}$ & $1,49.10^{6}$ & $6,64.10^{7}$ & $0 \mathrm{~d} 00: 00: 01$ & $0 \mathrm{~d} 00: 00: 32$ \\
\hline $10^{-6}$ & $1,49.10^{5}$ & $6,64.10^{6}$ & $0 \mathrm{~d} 00: 00: 00$ & $0 \mathrm{~d} 00: 00: 03$ \\
\hline
\end{tabular}

\section{Q - Factor}

Q-factor characterizes the quality of a digital signal from an analog point of view, therefore it is judged as a signal / noise ratio. In practical measurements can be determined the difference of a signal level from a noise level and subsequently estimated the parameters such as the error rate and Q - factor. [3][4]

The distance of the signal level to noise level can be determined from the following equation.

$$
O S N R=10 \log \left(\frac{P_{i}}{N_{i}}\right)+10 \log \left(\frac{B_{m}}{B_{r}}\right)
$$

$P_{i}$ - average power of the optical signal i-th channel $[\mathrm{W}]$

$N_{i}$ - interpolated value of the average noise power [W]

$B_{m}-$ spectral width, in which we measure [nm]

$B_{r}$ - reference bandwidth

Q-factor can be calculated from the following equation.

$$
Q=\frac{i_{H}-\gamma_{o p t}}{\sigma_{i H}}=\frac{\gamma_{o p t}-i_{L}}{\sigma_{i L}}
$$

$\gamma_{\text {opt }}$ - optimal value of the decision level

$i_{L}-$ current corresponding to the level of optical power on the photo detector for level log. 0 $i_{H}-$ current corresponding to the level of optical power on the photo detector for level log. 1

By excluding $\gamma_{o p t}$ from the equation (8) we obtain the relation:

$$
Q=\frac{i_{H}-i_{L}}{\sigma_{1 H}+\sigma_{1 L}}
$$

$\gamma_{o p t}$ - optimal value of the decision level 
$i_{H}, i_{L}$ - current corresponding to the level of optical power on the photo detector for levels log. 1 and log. 0

\section{Relationship Between Q-Factor And The BIT Error Rate}

Q-factor is a parameter that measures the quality of DWDM systems. These systems are characterized by the transmitting reliability of a large volume of data with a low error rate. According to these facts, the measurement of classical error would be very time consuming as shown in Table 2 .

\begin{tabular}{|c|c|c|c|c|c|}
\hline BER & $10^{-12}$ & $10^{-13}$ & $10^{-14}$ & $10^{-15}$ & $10^{-16}$ \\
\hline STM-16/OC-48 & $7 \mathrm{~min}$ & $70 \mathrm{~min}$ & $11 \mathrm{hrs}$ & 6 days & 46 days \\
\hline STM-64/OC-192 & $2 \mathrm{~min}$ & $17 \mathrm{~min}$ & $3 \mathrm{hrs}$ & $28 \mathrm{hrs}$ & 12 days \\
\hline \multicolumn{6}{|c|}{$\mathrm{Q}-$ factor $<1$ minute } \\
\hline
\end{tabular}

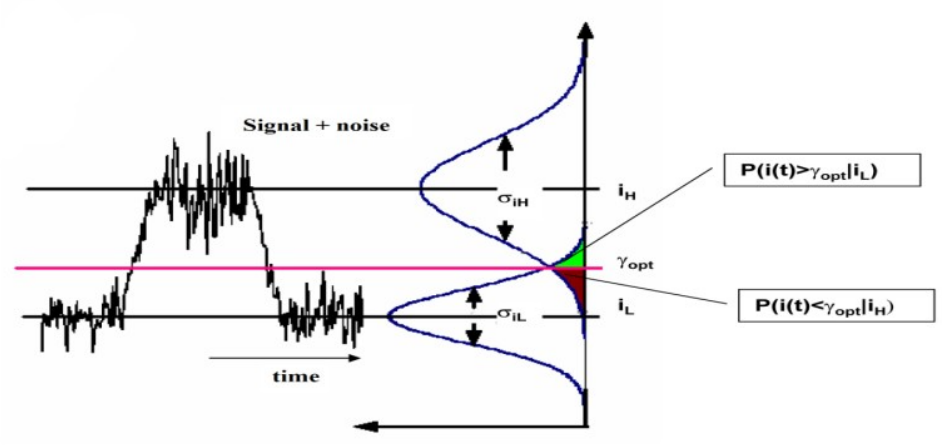

Figure $1 \mathrm{Q}$ - factor of the digital signal

Assuming that the occurrence probability of ones and zeros is the same, the relationship between Q-factor and the error rate can be expressed as follows. [5]

$$
B E R=P(\text { Error })=\frac{1}{\sqrt{2 \pi \sigma_{i L}^{2}}} \int_{\gamma_{o p t}}^{\infty} e^{-\frac{1}{2}\left(\frac{i-i_{L}}{\sigma_{i l}}\right)^{2}} d i=\operatorname{erfc}(Q)
$$

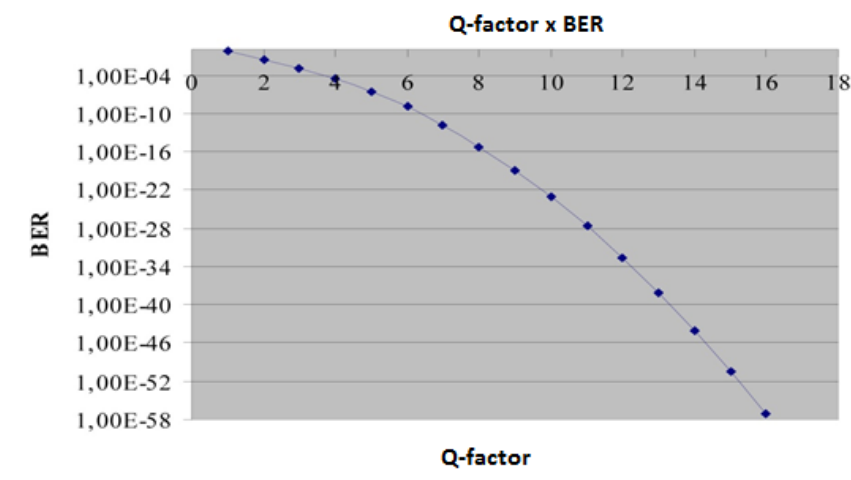

Figure 2 Relationship between Q-factor and the bit error rate

\section{Conclusion}

In backbone networks dominate optical fibers and further development is going through DWDM wavelength multiplexing. The purpose of the optical hierarchy is to create a common platform for different types of networks to support advanced service parts. In this article we have tried to explain the connection between the individual error parameters in optical wireless networks and nodes, which leads to the conversion of the optical signal to an electrical signal. The article describes the relationship between Q-factor and the bit error rate. The speed measurement, independence of the digital signal structure and a wide range of transmitted speeds predispose Q-factor for the monitoring of DWDM systems.

\section{References}

[1] P. Ivaniga, Evaluation of error rate in high-speed digital networks, Published by University of Žilina in Žilina, EDIS - ŽU 2007, $87 \mathrm{p}, 1^{\text {st }}$ Edition, ISBN 978-80-8070-771-2.

[2] P. Tošovský, Tester for optical fiber-free connection, Elektrorevue 53, 2007, ISSN 1213-1539, p. 1-6.

[3] L. Mikuš, Evaluations of the error rate in backbone networks, Elektrorevue 2(12) 2010, ISSN 1213-1539 p.1-6.

[4] D. Kilper, et al., Optical Performance monitoring. IEEE Lightwave Technology Journal, 22, 2006 p. $294-304$.

[5] P. Ivaniga, Error rate characteristics for multi operator transmission path, Elektrorevue 1(15) 2013, ISSN 1213 -1539 